\title{
A Computational Study of the Diels-Alder Reactions Between 2,3-Dibromo-1,3-Butadiene and Maleic Anhydride ${ }^{\text {th }}$
}

\author{
Uxía Rivero, Markus Meuwly, Stefan Willitsch \\ Departament of Chemistry, University of Basel \\ Klingelbergstrasse 80, 4056 Basel Switzerland
}

\begin{abstract}
The neutral and cationic Diels-Alder-type reactions between 2,3-dibromo-1,3-butadiene and maleic anhydride have been computationally explored as the first step of a combined experimental and theoretical study. Density functional theory calculations show that the neutral reaction is concerted while the cationic reaction can be either concerted or stepwise. Further isomerizations of the Diels-Alder products have been studied in order to predict possible fragmentation pathways that could happen in gas-phase experiments. Rice-Ramsperger-Kassel-Marcus (RRKM) calculations suggest that under single-collision experimental conditions the neutral product may reform the reactants and the cationic product will most likely eliminate $\mathrm{CO}_{2}$.
\end{abstract}

Keywords:

Diels-Alder reaction, Maleic Anhydride, 2,3-Dibromo-1,3-Butadiene, DFT, RRKM

\section{Introduction}

The Diels-Alder reaction is one of the most important reactions for the synthesis of cyclic compounds in organic chemistry because of its high regio- and stereo-selectivity $[1,2]$. In this reaction, a diene reacts with a dienophile to form a cyclic product. Two $\sigma$ bonds and one $\pi$ bond are formed from three $\pi$ bonds as depicted in Scheme 1.

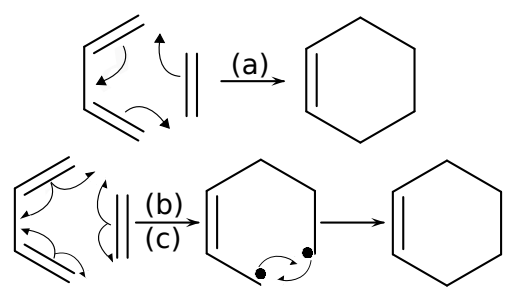

Scheme 1

This reaction has been the subject of many experimental and computational studies aiming to decide if it takes place in a concerted fashion and if so, whether or not it is a synchronous process and how the mechanism depends on the geometric and electronic properties of the reactants $[3,4,5,6,7,8,9,10,11$, $12,13,14]$.

The concertedness of a mechanism is determined by the topology of the potential energy surface (PES) [15]. A concerted mechanism occurs when the PES exhibits only one transition state (TS) between reactants and products so that the process takes place in a single step. A mechanism will be stepwise (taking place in two or more elementary steps) when the system

Email address: stefan.willitsch@unibas.ch (Stefan Willitsch) has to overcome at least two TSs separated by an intermediate species (a local minimum on the PES) to evolve from reactants to products.

Synchronicity refers to the time elapsed between the formation of the first and the second bond [15]. It is usually defined by the TS geometry [4]. Symmetric TSs give rise to synchronous processes in which both bonds are formed at the same time. Asymmetric TSs lead to asynchronous processes in which first one bond is formed and then the second follows. This definition of synchronicity has recently been questioned by some authors who argue that synchronicity should not be defined from a geometric but from a dynamic point of view because the connection between spatial quantities and temporal concepts might not always be valid [5]. A synchronous process is always concerted while an asynchronous one can be concerted or stepwise depending on the absence of a (stable) intermediate.

There has been a long-standing discussion about synchronicity and concertedness of Diels-Alder reactions which is not yet resolved $[3,6]$. The picture that organic chemistry textbooks usually give is that it is a concerted, synchronous reaction governed by the Woodward-Hoffmann rules that involves an aromatic TS $[2,16]$. However, experiments and calculations show that this is not that simple in many cases. In principle, one can think of three possible mechanisms (see Scheme 1): (a) synchronous concerted, (b) stepwise with a short lived intermediate whose lifetime is not long enough for the system to rotate around a $\mathrm{C}-\mathrm{C}$ bond, and (c) stepwise with a long lived intermediate. Note that when the system cannot rotate around a C-C bond, as is the case in (a) and (b), the reaction is stereoselective and only the s-cis conformer of the diene will yield the cyclic Diels-Alder product. On the contrary, mechanism (c) 
is not stereo-selective and the s-trans conformer of the diene could also in principle yield a Diels-Alder product.

Zewail and his group have performed different experiments involving retro Diels-Alder reactions in which they detected intermediates $[7,8]$. This suggests that the reaction must happen, at least partially, in a non-concerted fashion in these cases. However, since the experiment is started with the excitation of the Diels-Alder product, excited states play a role in the dynamics [17]. There also exist several studies involving kinetic isotope effects that compare experimental and calculated results in order to determine if the reaction is concerted or stepwise. The picture emerged that neutral Diels-Alder reactions tend to take place in a concerted way while ionic Diels-Alder reactions usually happen in a stepwise manner $[9,10,11]$.

From a computational point of view, studying Diels-Alder reactions is challenging since the relevant systems are comparatively large and the choice of method and basis set will bias the final result $[18,19,20]$. The reaction between butadiene and ethene (the simplest Diels-Alder reaction), see scheme 1, has been studied at various levels of theory [3, 12, 21, 22, 23]. For this symmetric reaction, ab initio and density functional theory (DFT) calculations favor a concerted, synchronous mechanism (as suggested by the symmetry of the system), but multireference methods are needed to accurately calculate the activation energy and the enthalpy of reaction as compared to the experimental values [18, 21, 22]. DFT methods can also provide good results, but due to the wide range of resulting energies the choice of functional is not trivial $[22,23]$.

The complexity is higher when studying asymmetric reactions due to the fact that a continuous range of possibilities exists between synchronous, concerted processes and asynchronous, stepwise mechanisms [13]. Moreover, there seems to be a correlation between the asymmetry of the TS structure and the rate constant of the process, where asymmetry refers to the difference in the lengths of the newly formed $\sigma$ bonds in the TS. The more asymmetric the TS geometry, the faster the reaction $[4,13]$. Diels-Alder reactions are usually activated by electron-withdrawing groups in the dienophile and electron-rich groups in the diene although the contrary situation, in which the electron-rich groups are in the dienophile and the electron-withdrowing ones in the diene, is also possible [2].

In general and compared to experimental values and high level theoretical calculations, Møller-Plesset second order perturbation theory (MP2) was found to underestimate the activation energies because of an overestimation of electron correlation [22]. Complete active space self consistent field (CASSCF) calculations give values close to Hartree-Fock (HF) due to its lack of dynamic correlation, which causes an overestimation of the height of the reaction barrier [21, 22]. It is thus necessary to use complete active space second order perturbation theory (CASPT2) to obtain accurate energies [22]. On the other side, DFT methods predict a wide range of activation energies and reaction enthalpies and the widely used B3LYP/6-31G* approach usually overestimates the activation energies while underestimating the reaction enthalpies [20, 23, 24]. Moreover, it seems that increasing the basis set does not improve the quality of the results and it can even make them worse. A medium-size basis set, such as 6-31G*, has thus been recommended for the study of Diels-Alder reactions [19, 24, 25].

Cationic Diels-Alder reactions (polar cycloadditions) are often faster but still show a high degree of stereoselectivity $[26,27,28]$. There have been some studies on the conservation of orbital symmetry to try to construct rules analogous to the Woodward-Hoffmann rules widely used for the neutral reactions [28, 29, 30]. Wiest and Donoghe proposed a model in which the electronic state symmetry must be conserved throughout the reaction [14]. Gas-phase experiments on the cationic Diels-Alder reaction between butadiene and ethene have been unable to isolate the Diels-Alder product. As no efficient deactivation was possible in the gas phase, the product fragmented under the experimental conditions [31]. Subsequent computational studies explored the possible fragmentation pathways of the Diels-Alder product in order to interpret the experimental findings $[32,33]$.

In summary, these results corroborate the picture that neutral reactions usually occur in a concerted fashion while in cationic systems a non-concerted mechanism is favored [3, 14]. However, the border between asynchronous, concerted and stepwise mechanisms is not yet clear [9, 10, 13]. It has also been argued that both concerted and stepwise mechanisms can be present at the temperatures at which these reactions are usually performed (around $500 \mathrm{~K}$ and above) due to the energetic proximity of both pathways in many systems $[5,7,8]$. When a stepwise process takes place, the competition between the closure of the ring and the isomerization of the intermediate state needs to be studied in order to determine whether the reaction will be stereo-selective.

From an experimental perspective, the most precise data on reaction mechanisms and dynamics can be gained from gasphase studies performed under single-collision conditions. As the progress in molecular-beam experiments allows the probing ever-larger systems under precisely defined conditions [34], the open questions pertaining to the mechanistic details of DielsAlder reactions become an attractive target of study. However, a crucial difference to the solution phase, for which the vast majority of experimental data is available, is that the energy released into the products over the course of the reaction cannot be quenched by the environment under single-collision conditions on the timescales of experiments and is therefore available to drive consecutive fragmentations or isomerizations. This effect is particularly important for Diels-Alder processes in which the total energy release of the reaction remains locked in a single product. As only scarce information is available on these important mechanistic aspects [35], we present here a comprehensive theoretical characterization of the mechanisms, decay pathways and kinetics of the products of the neutral and ionic Diels-Alder reaction between 2,3-dibromo-1,3-budadiene (DBB) and maleic anhydride (MA) which highlights these effects and may serve as a guide to future experiments.

We have chosen DBB as the diene because it is a generic, activated diene which fulfills the experimental requirements for conformational separation of its isomers by electrostatic deflection of a molecular beam [34] thus enabling the characterization of conformational aspects and specificities of the reaction. MA 
is a widely used, activated dienophile which due to its symmetry simplifies the possible outcomes of the reaction. The reaction of DBB and MA thus serves as a prototypical system well suited to explore general mechanistic aspects of Diels-Alder processes under gas-phase conditions.

In this paper, we present computational results on this system using DFT and multi-reference (CASPT2) approaches (Section 3.1). We also study the evolution of the Diels-Alder products by exploring different isomerization and fragmentation pathways (Section 3.2) based on RRKM theory [36] in order to obtain qualitative trends for the time evolution of the system under collisionless conditions (Section 3.3).

\section{Methods}

The PES of the system was calculated at the DFT level of theory with the M06-2X functional [37] as recommended by Linder and Brinck [20] and using the Gaussian09 suite of codes [38]. The 6-31G* basis set was used as recommended in Refs. $[19,24]$. We have employed the GRRM14 program [39] to scan the PES for stationary points by performing a global reaction route mapping (GRRM) calculation [40] at the PM6 level of theory [41] to investigate the isomerization and fragmentation pathways of the products of the Diels-Alder reactions. The stationary points found both by GRRM and by manual exploration of the PES have been optimized at the B3LYP/6-31G* level [42] and then reoptimized at the M06-2X/6-31G* level of theory. All the geometry optimizations have been followed by frequency calculations and the connectivities of the TSs with the minima have been checked by means of internal reaction coordinate (IRC) calculations [43]. The energies have been corrected for zero-point vibrational energies without scaling the frequencies. The optimized geometries (in Cartesian coordinates) of all the structures are given in the supplementary information. Single point calculations of some of the stationary points at the CASPT2 $(5,5) /$ aug-cc-pVDZ level of theory as implemented in Molcas 8.0 [44] were performed.

We have employed RRKM theory in combination with a master equation approach (equation (1)) to follow the time evolution of the products of the Diels-Alder reactions until their eventual fragmentation [36].

$$
-\frac{d P_{i}(t)}{d t}=\sum_{j \neq i} k_{i j}(E) P_{i}(t)-\sum_{l \neq i} k_{l i}(E) P_{l}(t)
$$

$P_{i}(t)$ is the population of species $i$ at time $t$ and $k_{i j}(E)$ is the microcanonical RRKM rate for the formation of species $j$ from species $i$ at energy $E$. The different $k_{i j}(E)$ have been calculated using Multiwell [45]. The frequencies used in these calculations are given in the supplementary information. Tunneling has been taken into account using an asymmetric Eckart barrier model [46]. We have used Maple 2015 [47] with the Rosenbrock numerical method for solving equation (1) [48].

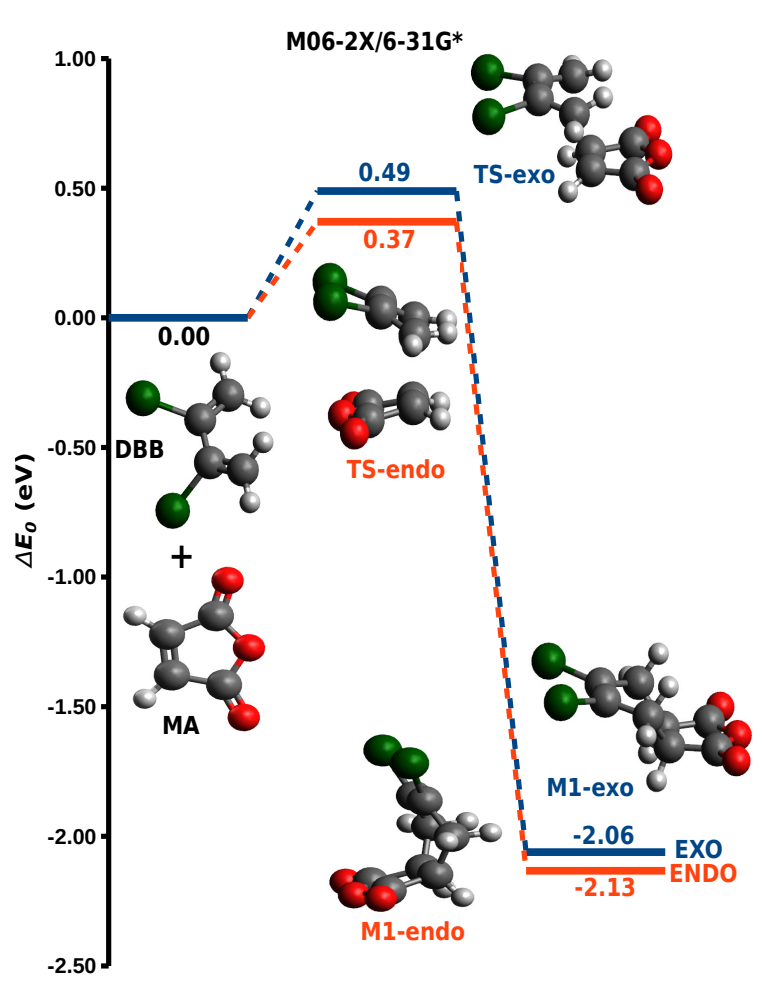

Figure 1: Potential energy surface for the two possible neutral Diels-Alder reactions (exo in blue and endo in orange) between s-cis-2,3-dibromo-1,3-butadiene (s-cis-DBB) and maleic anhydride (MA) at the M06-2X/6-31G* level of theory. The relative energies in $\mathrm{eV}$ with respect to the reactants as well as the structures of minima and transition states are shown. Green spheres represent bromine atoms, red spheres oxygen atoms, gray spheres carbon atoms and white spheres hydrogen atoms.

\section{Results and Discussion}

\subsection{Diels-Alder reaction}

Figures 1 and 2 show stationary points along the reaction coordinate for the neutral and cationic Diels-Alder reactions between DBB and MA at the M06-2X/6-31G* level of theory. The two surfaces calculated at the B3LYP/6-31G* level of theory can be found in the supplementary material (Figures $\mathrm{S} 1$ and $\mathrm{S} 2$ ). Because both reactant molecules are symmetric, there are two possible paths for the Diels-Alder reaction to follow. These are referred to as "endo" and "exo" depending on the relative orientation of the reactants. M06-2X clearly favors the endo path over the exo as expected for this kind of reactions [49].

Judging from the PESs and the geometries of the TSs, the neutral reaction (Figure 1) is concerted and symmetric. This is not a surprise since, even though s-cis-DBB is not planar, it becomes planar when it interacts with MA making the TSs symmetric.

The situation for the cationic case is quite different (Figure 2). With an odd number of $\pi$ electrons, the symmetry is broken and this is no longer a [4+2] but a [3+2] cycloaddition so the TSs are now asymmetric. Interestingly, the endo path is predicted to be concerted while the exo path is so asymmetric that it switches to a stepwise mechanism with a shallow intermediate between the two TSs. Because a stepwise mechanism 
(a)

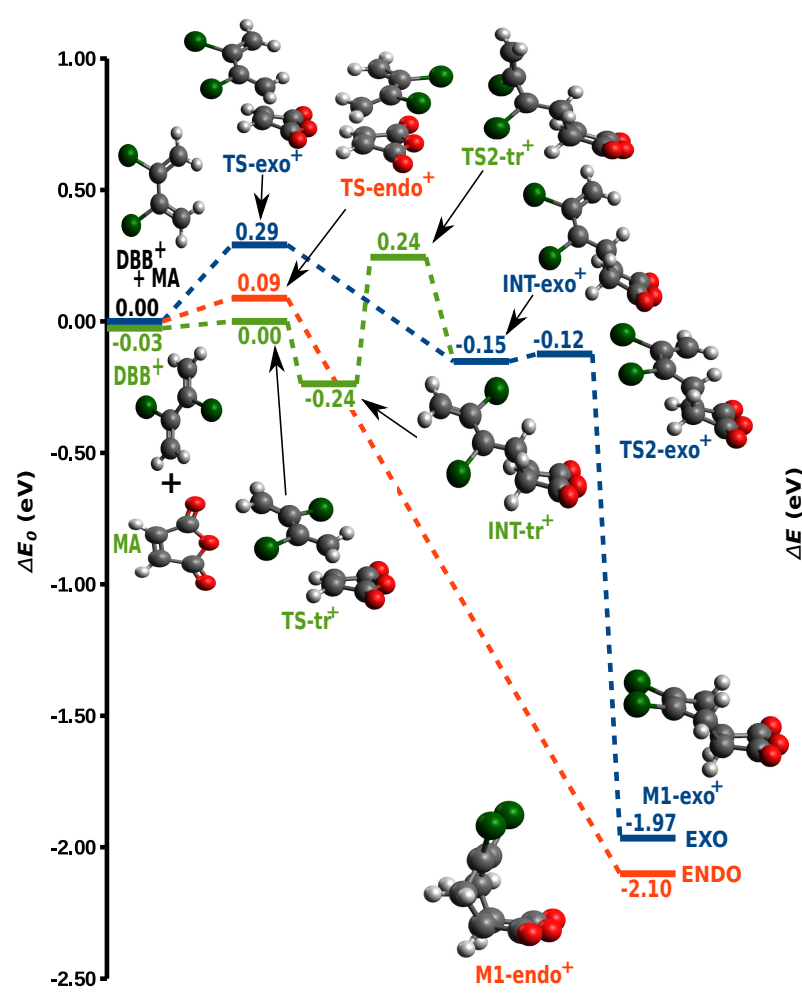

(b)

M06-2X/6-31G* (c)

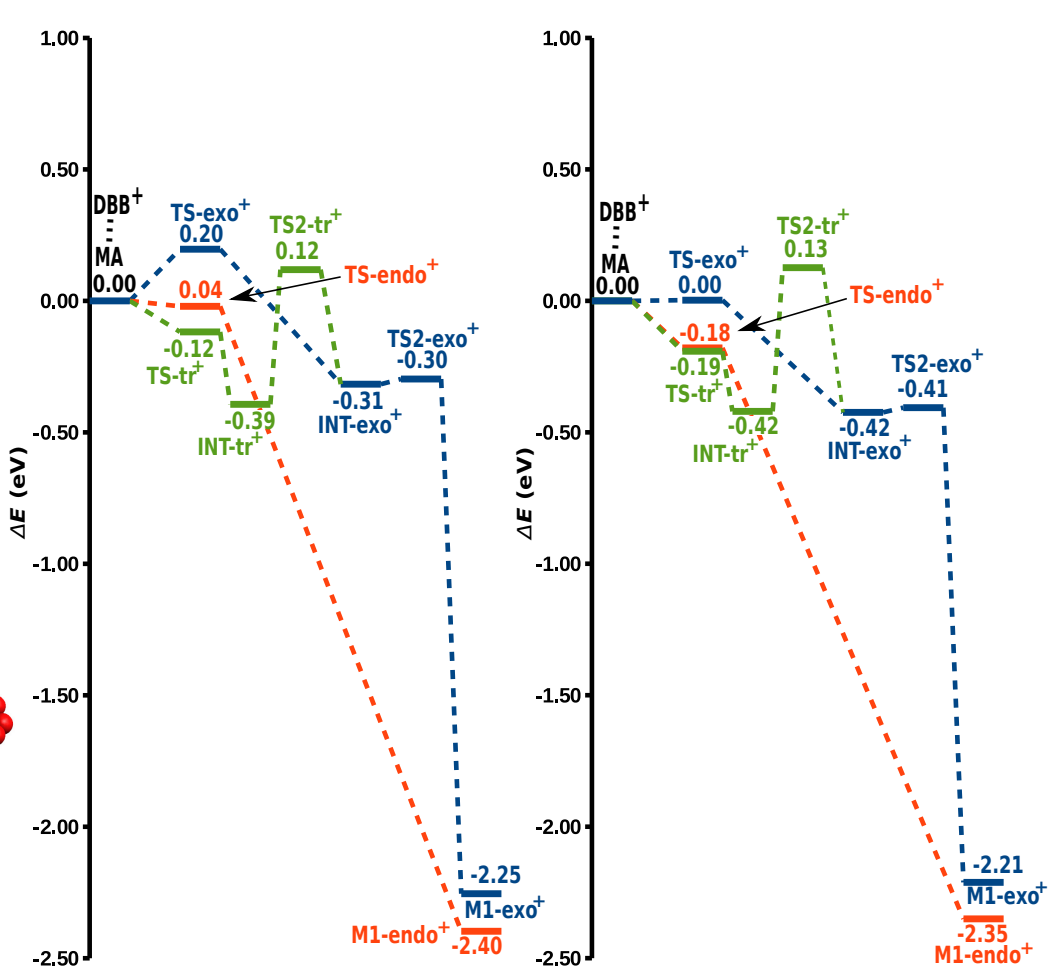

Figure 2: Potential energy surface for the three possible cationic Diels-Alder reactions (exo in blue, endo in orange and with the s-trans conformer in green) between cationic 2,3-dibromo-1,3-butadiene $\left(\mathrm{DBB}^{+}\right)$and maleic anhydride (MA). (a) Zero point corrected relative energies in eV with respect to the reactants at infinite distance at the M06-2X/6-31G* level of theory. The structures of minima and transition states are shown. Green spheres represent bromine atoms, red spheres oxygen atoms, gray spheres carbon atoms and white spheres hydrogen atoms. (b) and (c) Relative electronic energies in eV with respect to the reactants (with $\mathrm{DBB}^{+}$ in its s-cis conformation) at a distance of $7 \AA$ at the M06-2X/6-31G* and CASPT2/aug-cc-pVDZ levels of theory respectively.

becomes favorable, the s-trans conformer of DBB is also reactive. In fact, we were able to locate one path (in green in Figure 2) connecting the reaction of the s-trans conformer with the intermediate of the exo path. In order to explore the multireference character of the cationic surface, single point calculations at the CASPT2 (5,5)/aug-cc-pVDZ level of theory were carried. Note that no zero point energy is being considered and that the zero of energy corresponds to the two reactant molecules (with DBB in its s-cis conformation) being $7 \AA$ apart instead of at infinite distance. It can be seen that CASPT2 largely agrees with M06-2X but the height of the barriers is reduced. We have also performed single point calculations at the MS(2)CASPT2(5,5)/aug-cc-pVDZ level of theory finding that the energetic difference between the ground state and the first excited state surface is around $1 \mathrm{eV}$ or higher at all the stationary points of Figure 2. This is also in line with a CCSD/aug-cc-pVDZ single point calculation that we performed for M1-endo (since it is the stationary point where the ground state surface and the first excited state surface approach each other the most) obtaining a value of the $\mathrm{T} 1$ diagnostic of 0.0207 . It is usually argued that values below 0.02 indicate that a single reference wave function is sufficient to treat a system while values above 0.02 indicate multireference character [50]. We therefore believe that the single-reference M06-2X/6-31G* method should be sufficient for our purposes, i.e., studying the qualitative aspects of the reactivity of this system. It is worth to realize that, in the exo path, the closure of the ring is predicted to have a lower activation barrier $(0.03 \mathrm{eV})$ than the isomerization into the strans intermediate $(0.39 \mathrm{eV})$. Thus, a stereo-selective reaction should be favored even if the mechanism is stepwise. Further dynamic (and experimental) studies are needed in order to confirm this hypothesis. The activation barriers in the cationic reaction $(0.09 \mathrm{eV}$ and $0.29 \mathrm{eV})$ are much lower than those in the neutral system $(0.37 \mathrm{eV}$ and $0.49 \mathrm{eV})$ implying faster kinetics for the ionic variant.

\subsection{Isomerizations and fragmentations of the Diels-Alder products}

We are interested in the possible isomerizations and fragmentations of the Diels-Alder products to explore their reactivity under typical gas-phase single-collision conditions. Subsequent reactions which have been identified are shown in Figures 3 and 4. Note that only one backward reaction was considered because, with the energy available in the system after the formation of the Diels-Alder product, the endo and exo product species which are conformers (see the schemes of the relevant molecular geometries in Figures S3 and S4) can freely interconvert. Additionally, in the construction of the energy profiles, we only considered structures with different atom connectivities to be different minima. For this reason, rotational and conforma- 


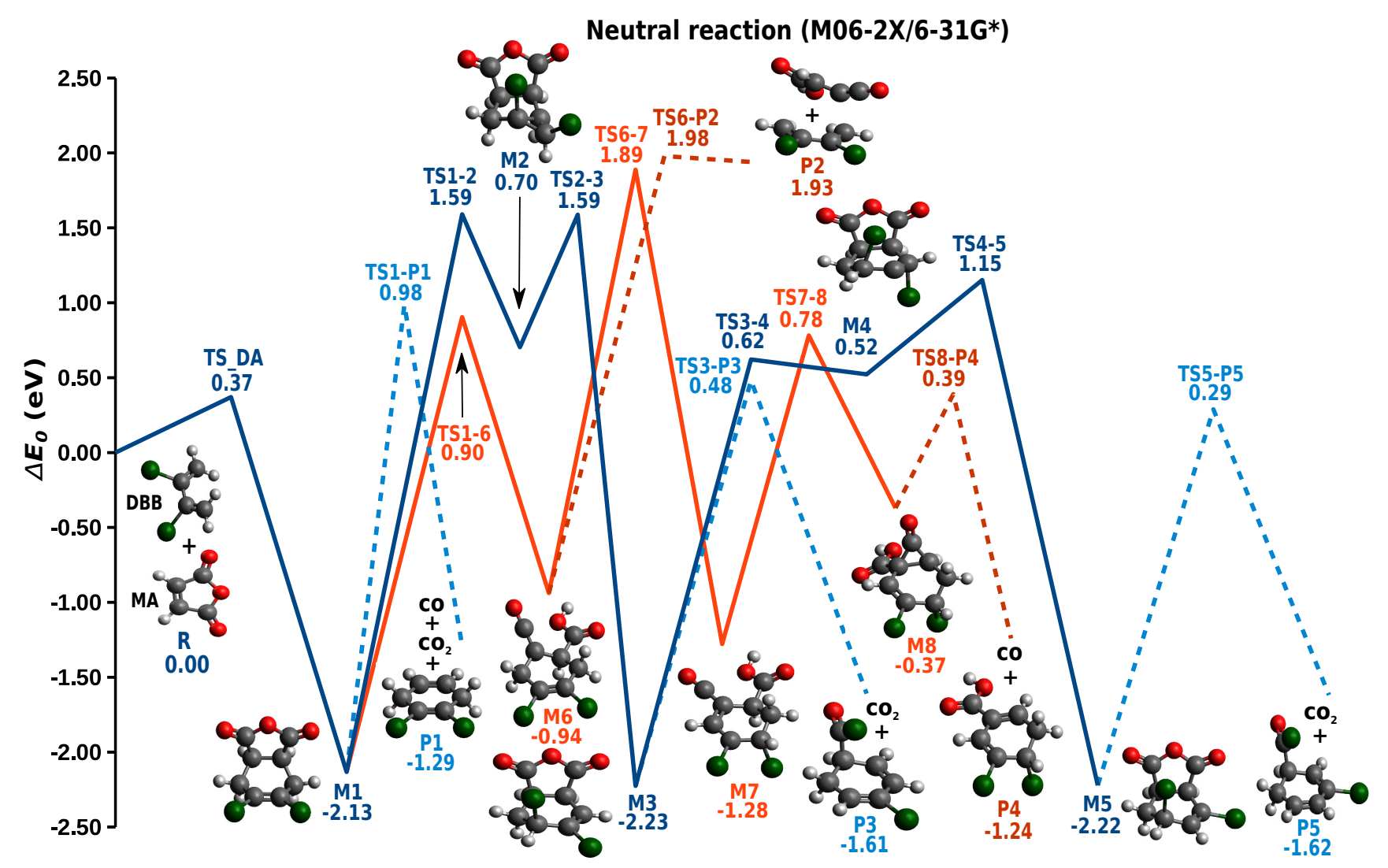

Figure 3: Energy profiles at the M06-2X/6-31G* level of theory for some of the possible isomerizations and fragmentations of the neutral Diels-Alder product. The structures of the different minima are shown. Dashed lines indicate dissociations and solid blue and orange lines two possible isomerization pathways. Green spheres represent bromine atoms, red spheres oxygen atoms, gray spheres carbon atoms and white spheres hydrogen atoms.

tional isomers are not distinguished here. We chose the most stable structure among the ones we found to represent each family of isomers. Finally, we only show the most favorable dissociation from each minimum.

The product of the neutral Diels-Alder reaction (M1) can undergo a dissociation into $\mathrm{CO}+\mathrm{CO}_{2}+\mathrm{P} 1$ via TS1-P1 or two kinds of isomerizations (Figure 3): (a) in blue, migration of a hydrogen atom via TS1-2 and TS2-3 followed by migration of bromine via TS3-4 and another migration of hydrogen via TS45 or (b) in orange, migration of a hydrogen atom that breaks the 5-membered ring via TS1-6, followed by another hydrogen migration (TS6-7) and an -OH migration (TS7-8). Different isomers can eliminate $\mathrm{CO}$ (M8 via TS8-P4) or $\mathrm{CO}_{2}$ (M3 via TS3$\mathrm{P} 3$ and M5 via TS5-P5) or break into DBB and an open isomer of MA (M6 via TS6-P2). Note that all these paths from M1 have high activation barriers (TS1-P1: $3.11 \mathrm{eV}$; TS1-2: $3.72 \mathrm{eV}$ and TS1-6: $3.03 \mathrm{eV}$ ) and are predicted to be all less favorable than the backward Diels-Alder reaction (TS_DA: $2.50 \mathrm{eV}$ ).

The cationic Diels-Alder product $\mathrm{M}^{+}$can eliminate $\mathrm{CO}_{2}$ forming $\mathrm{P}^{+}$via TS1-P1 ${ }^{+}$or undergo two different isomerizations (Figure 4): (a) in blue, the migration of bromine and the transition from a 6-membered ring to a 5-membered ring via $\mathrm{TS} 1-2^{+}$to form $\mathrm{M}^{+}$followed by different bromine (TS2-3 $3^{+}$ and TS3-4 $4^{+}$), hydrogen (TS4-5 ${ }^{+}$and TS5-6 $6^{+}$) and -OH (TS6$7^{+}$) migrations ultimately leading to $\mathrm{M}^{+}$or $(\mathrm{b})$ in orange, a hydrogen atom migration (TS1-10 ${ }^{+}$) followed by a migration of bromine that causes the closure of the 6-membered ring into a 5membered ring via TS10-11 $1^{+}$and subsequent bromine (TS11$12^{+}$and $\mathrm{TS} 12-13^{+}$), hydrogen (TS13-14+, ${ }^{+}$S $14-5^{+}$, TS15$16^{+}$and $\mathrm{TS} 17-18^{+}$) and $-\mathrm{OH}\left(\mathrm{TS} 16-17^{+}\right)$migrations ultimately leading again to $\mathrm{M}^{+}$. In this case, both paths are interconnected. Note that Bouchoux et al. have already found a closure of a 6-membered ring into a 5-membered ring when they explored the evolution of cationic cyclohexene [33]. The system can eliminate $\mathrm{H}_{2}\left(\mathrm{TS}^{+}-\mathrm{P} 2^{+}\right)$and $\mathrm{HBr}\left(\mathrm{TS}^{+}-\mathrm{P} 3^{+}\right)$at different points along the isomerization paths $\left(\mathrm{M}^{+}, \mathrm{M} 9^{+}\right)$. In this case, the processes leading to the formation of $\mathrm{M}^{+} 0^{+}\left(E_{a}=0.98 \mathrm{eV}\right)$ and the dissociation into $\mathrm{CO}_{2}+\mathrm{P}^{+}\left(E_{a}=1.68 \mathrm{eV}\right)$ are predicted to be more favorable than the backward Diels-Alder reaction $\left(E_{a}=2.19 \mathrm{eV}\right)$.

The energy profiles of both the neutral and the cationic systems at the B3LYP level (Figures S5 and S6 of the supplementary information) qualitatively agree with the M06-2X picture.

Comparing the neutral and cationic profiles, it can be seen that the cationic activation barriers are much lower than the neutral ones so the kinetics should be faster in the cationic system. Contrary to the neutral, in the cationic case there are predicted to be two transition states with lower activation barriers than the backward Diels-Alder reaction leading to alternative decay routes of the Diels-Alder products in a gas-phase experiment. 


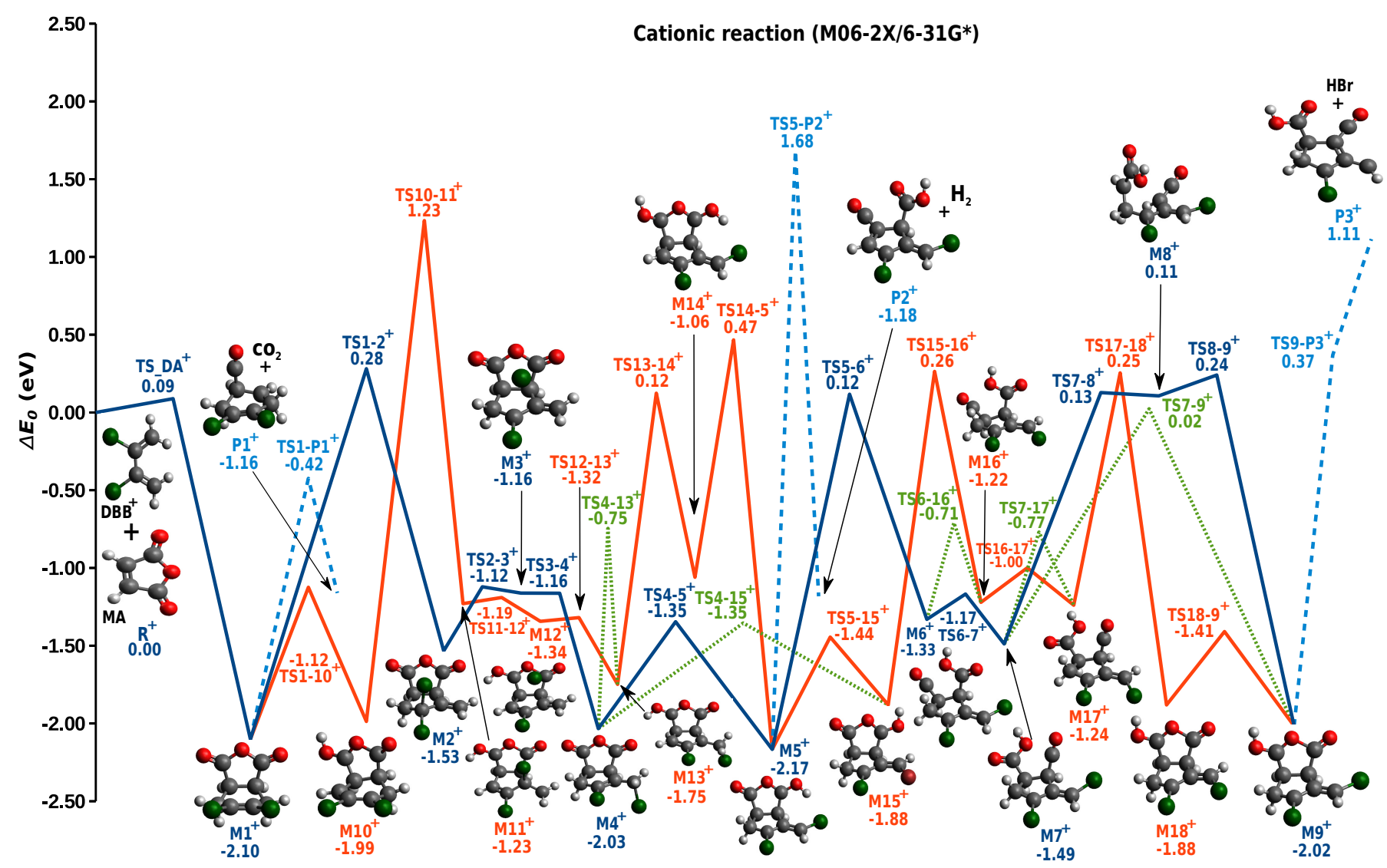

Figure 4: Energy profiles at the M06-2X/6-31G* level of theory for some of the possible isomerizations and fragmentations of the cationic Diels-Alder product. The structures of the different minima are shown. Dashed lines indicate dissociations, solid blue and orange lines two possible isomerization pathways and green lines connections between the two paths. Green spheres represent bromine atoms, red spheres oxygen atoms, gray spheres carbon atoms and white spheres hydrogen atoms.

\subsection{RRKM calculations}

The population of selected species along the previously discussed isomerization paths at different excess energies as a function of the logarithm of time are shown in Figures 5 and 7 for the neutral and cationic reactions, respectively. At $t=0 \mathrm{~s}$, the system is initialized in the product of the Diels-Alder reaction i.e. $\mathrm{M} 1$ for the neutral system and $\mathrm{M}^{+}$for the cationic one. The populations at each time are calculated by solving equation (1). Dissociation processes are treated as irreversible.

The RRKM calculations for the neutral system at $2.17 \mathrm{eV}$ of excess energy above the energy of the reactants (i.e. $\mathrm{DBB}+\mathrm{MA})$ is shown in Figure 5(a). We have chosen this particular energy because it is achievable under typical experimental conditions. It can be seen that with increasing time the reactants are re-populated which means that the backward reaction dominates the dynamics of M1 at this energy. There is also a small contribution of the dissociation into $\mathrm{P} 1+\mathrm{CO}+\mathrm{CO}_{2}$. The isomerization paths are found to be insignificant. This situation changes at double excess energy (4.34 eV, Figure 5(b)), where the kinetics are predicted to be four orders of magnitude faster than in Figure 5(a). There is now a considerable probability that the system dissociates into $\mathrm{P} 1+\mathrm{CO}+\mathrm{CO}_{2}$. Again, the backward reaction plays an important role and the isomerization paths barely participate.
Figure 6 shows the distribution of the population of the most important species at $t=1 \cdot 10^{-6} \mathrm{~s}$ as a function of the excess energy. This time corresponds to typical experimental timescales for the detection of reaction products in molecular-beam experiments. It can be seen that the product of the Diels-Alder reaction M1 is stable on the microsecond timescale at experimentally achievable energies (indicated in brown in Figure 6) and only at high excess energies dissociation products are obtained on this timescale. Even at the highest energies studied, no noticeable participation of the isomerization pathways was found.

The evolution of the cationic reaction at the energy of the reactants (i.e. $\mathrm{DBB}^{+}+\mathrm{MA}$ ) is shown in Figure 7(a). At this available energy, the open channels are: dissociation into

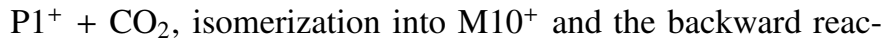
tion from $\mathrm{M} 10^{+}$to $\mathrm{M}^{+}$. We first see how part of the population of $\mathrm{M}^{+}$isomerizes into $\mathrm{M}^{+} 0^{+}$until an equilibration between the populations of the two species is reached. Due to the fact that the most favorable process to happen from $\mathrm{M} 1^{+}$is the isomerization into $\mathrm{M}^{+} 0^{+}$while the other possible transitions from $\mathrm{M}^{+}$and $\mathrm{M}^{+} 0^{+}$have much higher activation energies, the system is trapped in these two minima until it overcomes the barrier for dissociating into $\mathrm{P}^{+}+\mathrm{CO}_{2}$. Therefore, with this available energy and on typical timescales of experiments, both 

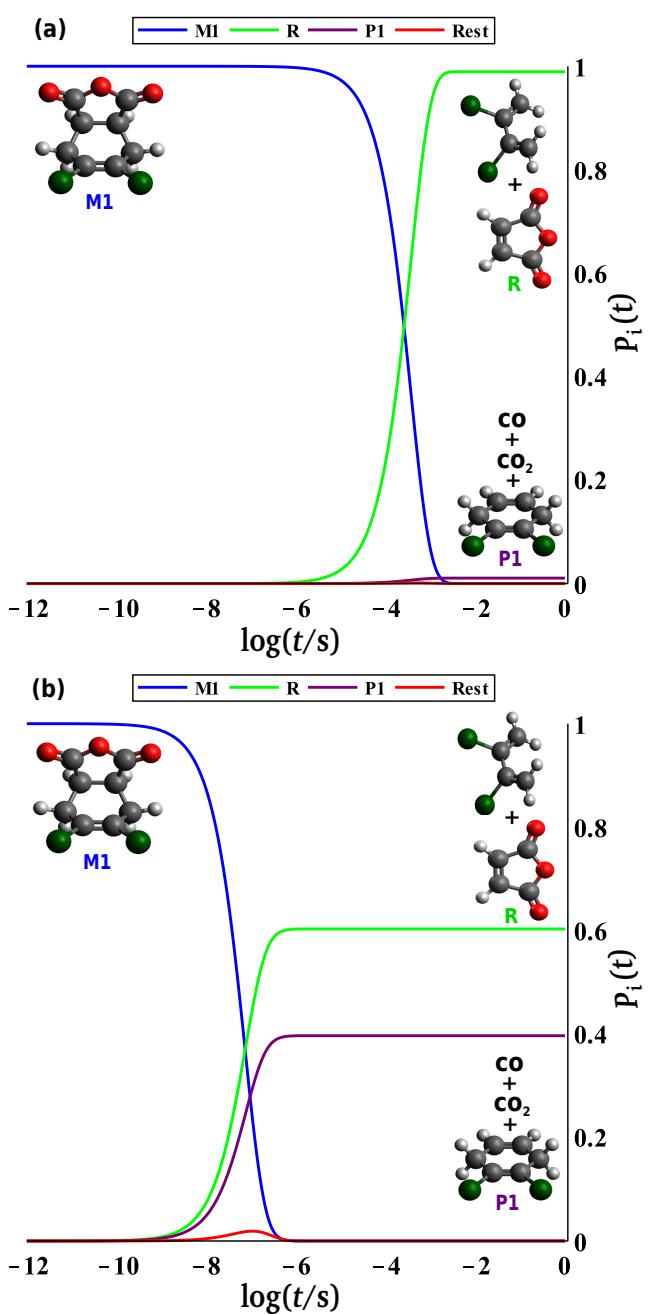

Figure 5: RRKM calculations for the neutral system with energies and frequencies calculated at the M06-2X/6-31G* level of theory at an energy of (a) $2.17 \mathrm{eV}$ and (b) $4.34 \mathrm{eV}$ above the energy of the Diels-Alder reactants assuming a microcanonical ensemble in a collisionless regime. Population of the most important species $\left(P_{i}(t)\right)$ versus logarithm of time. Rest corresponds to the sum of the populations of the species that are not explicitly shown. Green spheres represent bromine atoms, red spheres oxygen atoms, gray spheres carbon atoms and white spheres hydrogen atoms.

isomerization and dissociation into $\mathrm{P}^{+}+\mathrm{CO}_{2}$ of the cationic Diels-Alder product are predicted. The RRKM results of $\mathrm{M}^{+}$ at an excess energy of $1.97 \mathrm{eV}$ are displayed in Figure 7(b). This energy corresponds to the energy of the experimentally achievable configuration $\mathrm{DBB}+\mathrm{MA}^{+}$, i.e. the configuration in which the charge has initially been exchanged between the reactants relative to the energy of the reactants $\mathrm{DBB}^{+}+\mathrm{MA}$, at the M06-2X/6-31G* level. The reaction seems to be much faster than without excess energy. The time during which the system is trapped between $\mathrm{M}^{+}$and $\mathrm{M} 10^{+}$is now much shorter. The main channel is again the dissociation into $\mathrm{P}^{+}+\mathrm{CO}_{2}$, but there is now a small contribution of the backward DielsAlder reaction to the kinetics. At this energy, dissociation into $\mathrm{P}^{+}+\mathrm{CO}_{2}$ is observed on the nanosecond timescale which should be detectable in experiments. Note, however, that the asymptote $\mathrm{DBB}+\mathrm{MA}^{+}$corresponds to an excited state of the

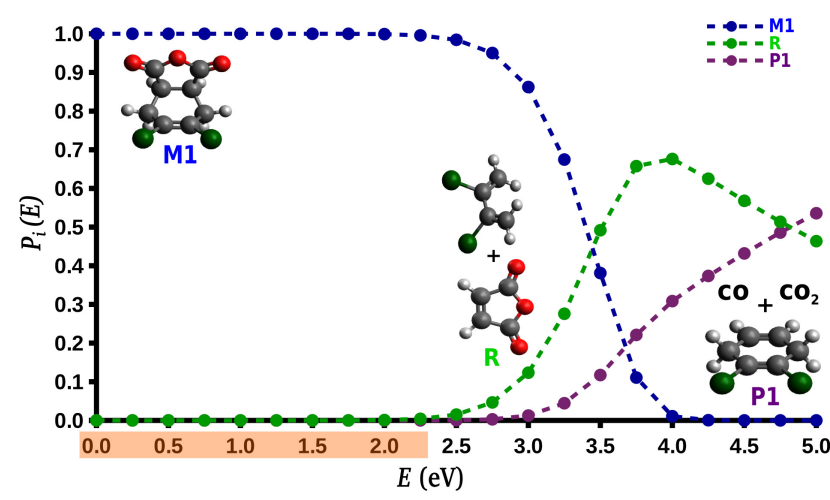

Figure 6: RRKM calculations for the neutral system with energies and frequencies calculated at the M06-2X/6-31G* level of theory at $t=1 \cdot 10^{-6} \mathrm{~s}$. Colored dots represent the population of the most important species $\left(P_{i}(E)\right)$ versus excess energy in $\mathrm{eV}$ above the energy of the reactants. The dashed lines help to guide the eye. Green spheres represent bromine atoms, red spheres oxygen atoms, grey spheres carbon atoms and white spheres hydrogen atoms. The brown rectangle indicates typical achievable energies in molecular-beam experiments.

system so excited-state dynamics may play a significant role in this case.

To further explore the reactivity of $\mathrm{M}^{+}$, Figure 8 shows how the populations of the different species in Figure 4 change as a function of excess energy at $t=1 \cdot 10^{-3} \mathrm{~s}$ which corresponds to a typical experimental timescale for reaction experiments with ions in traps. It can be seen that at low energies, the isomerization into $\mathrm{M}^{+} 0^{+}$is the dominant reaction of $\mathrm{M}^{+}$. At increasing energy, the main decay channel is the dissociation into $\mathrm{P}^{+}+\mathrm{CO}_{2}$. At the highest energies studied, the backward Diels-Alder reaction starts to participate.

\section{Conclusions}

We have studied the neutral and cationic Diels-Alder reactions between DBB and MA at DFT level. The neutral reaction is predicted to be concerted and symmetric while the cationic reaction is asymmetric and can either be concerted or stepwise. The s-trans conformer of DBB can contribute to the cationic stepwise reaction. We have also explored the time evolution of possible products of these reactions until dissociation under typical experimental conditions. The neutral system has higher activation barriers than the cationic system. We have employed RRKM theory finding that the kinetics of the cationic system is much faster than the neutral system. The neutral product is predicted to most likely return to the reactants on a timescale of hundreds of microseconds at an excess energy of $2.17 \mathrm{eV}$, which corresponds to an achievable excess energy in experiments. On the other hand, the cationic product is expected to eliminate $\mathrm{CO}_{2}$ on a nanosecond timescale at an excess energy of $1.97 \mathrm{eV}$, which approximately corresponds to the conditions in which MA is asymptotically the cationic species.

As a next step, we aim to explore the formation of the Van der Waals complexes between DBB and MA and their influence on the dynamics of the neutral reaction. 
(a)

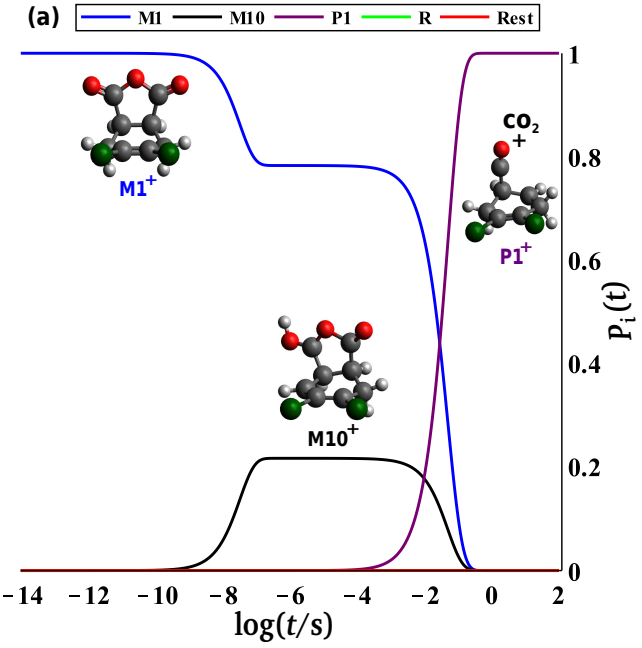

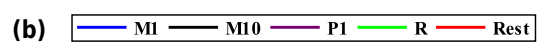

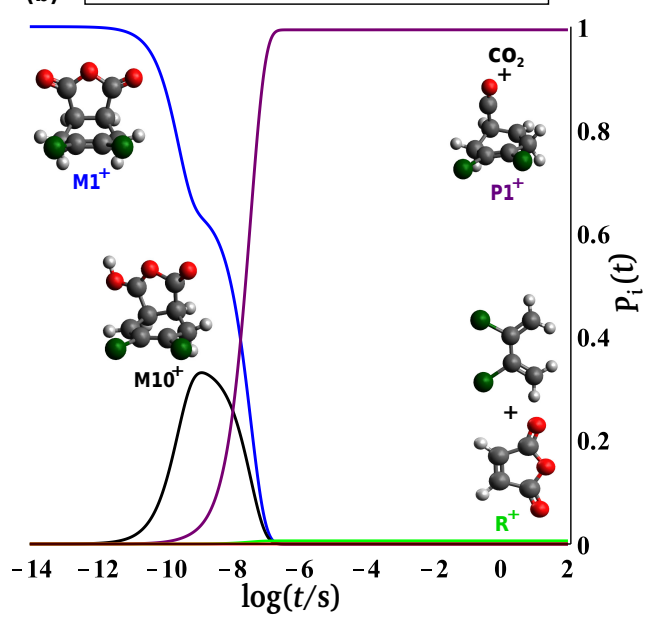

Figure 7: RRKM calculations for the cationic system with energies and frequencies calculated at M06-2X/6-31G* level of theory at an energy (a) equal to $0.00 \mathrm{eV}$ and (b) equal to $1.97 \mathrm{eV}$ above the energy of the Diels-Alder reactants assuming a microcanonical ensemble in a collisionless regime. Population of the most important species $\left(P_{i}(t)\right)$ versus logarithm of time. Rest corresponds to the sum of the populations of the species that are not explicitly shown. Green spheres represent bromine atoms, red spheres oxygen atoms, grey spheres carbon atoms and white spheres hydrogen atoms.

To our knowledge, this specific system had not been studied until now. Gaining insight into the differences between the cationic and neutral systems is crucial to understand the enhanced selectivity and rates of the cationic Diels-Alder reactions compared to their neutral counterparts. The present results may serve to design single-collision experiments that will probe these reactions and aid in their interpretation.

\section{Acknowledgements}

We thank Prof. O. Anatole von Lilienfeld for useful discussions. This work was supported by the Swiss National Science Foundation under grant nr. BSCGI0_157874.

\section{References}

[1] O. Diels, K. Alder, Justus Liebigs Ann. Chem. 460 (1928) 98.

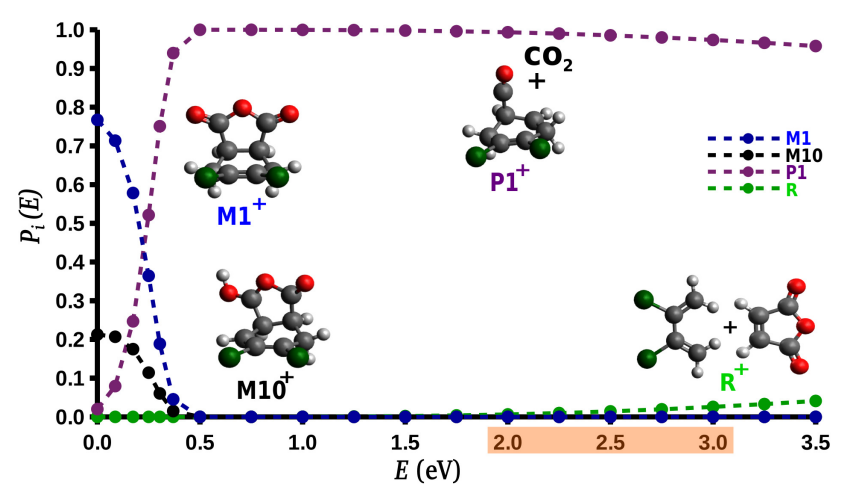

Figure 8: RRKM calculations for the cationic system with energies and frequencies calculated at M06-2X/6-31G* level of theory at $t=1 \cdot 10^{-3} \mathrm{~s}$. Colored dots represent the population of the most important species $\left(P_{i}(E)\right)$ versus excess energy in $\mathrm{eV}$ above the energy of the reactants and the dashed lines are for eye guidance. Green spheres represent bromine atoms, red spheres oxygen atoms, grey spheres carbon atoms and white spheres hydrogen atoms. The brown rectangle indicates typical experimentally achievable energies assuming the charge is initially located on MA.

[2] K. Ishihara, A. Sakakura, Comprehensive Organic Synthesis, 2nd Edition, Vol. 5, Elsevier, Oxford, 2014.

[3] K. N. Houk, J. González, Y. Li, Acc. Chem. Res. 28 (1995) 81.

[4] D. Yepes, O. Donoso-Tauda, P. Pérez, J. S. Murray, P. Politzer, P. Jaque, Phys. Chem. Chem. Phys. 15 (2013) 7311.

[5] M. A. F. de Souza, E. Ventura, S. A. do Monte, J. M. Riveros, R. L. Longo, J. Comput. Chem. 37 (2016) 701.

[6] L. R. Domingo, J. Chil. Chem. Soc. 2615 (2014) 59.

[7] B. A. Horn, J. L. Herek, A. H. Zewail, J. Am. Chem. Soc. 118 (1996) 8755.

[8] E. W.-G. Diau, S. De Feyter, A. H. Zewail, Chem. Phys. Lett. 304 (1999) 134.

[9] N. J. Saettel, O. Wiest, D. A. Singleton, M. P. Meyer, J. Am. Chem. Soc. 124 (2002) 11552.

[10] D. A. Singleton, B. E. Schulmeier, C. Hang, A. A. Thomas, S.-W. Leung, S. R. Merrigan, Tethraedron 57 (2001) 5149.

[11] E. Goldstein, B. Beno, K. N. Houk, J. Am. Chem. Soc. 118 (1996) 6036.

[12] S. Sakai, J. Phys. Chem. A 104 (2000) 922.

[13] L. R. Domingo, J. A. Sáez, Org. Biomol. Chem. 7 (2009) 3576.

[14] P. J. Donoghue, O. Wiest, Chem. Eur. J. 12 (2006) 7018.

[15] V. I. Minkin, Pure Appl. Chem. 71 (1999) 1919.

[16] R. Hoffmann, R. B. Woodward, Acc. Chem. Res. 1 (1968) 17.

[17] S. Wilsey, K. N. Houk, A. H. Zewail, J. Am. Chem. Soc. 121 (1999) 5772.

[18] H. Lischka, E. Ventura, M. Dallos, Chem. Phys. Chem. 5 (2004) 1365.

[19] G. Gayatri, Indian J. Chem. 50A (2011) 1579.

[20] M. Linder, T. Brinck, Phys. Chem. Chem. Phys. 15 (2013) 5108.

[21] O. Wiest, D. C. Montiel, K. N. Houk, J. Phys. Chem. A 101 (1997) 8378.

[22] V. Guner, K. S. Khuong, A. G. Leach, P. S. Lee, M. D. Bartberger, K. N. Houk, J. Phys. Chem. A 107 (2003) 11445

[23] P. D. Mezei, G. I. Csonka, M. Kállay, J. Chem. Theory Comput. 11 (2015) 2879.

[24] D. H. Ess, K. N. Houk, J. Phys. Chem. A 109 (2005) 9542.

[25] S.-Y. Tang, J. Shi, Q.-X. Guo, Org. Biomol. Chem. 10 (2012) 2673.

[26] D. J. Bellville, N. L. Bauld, J. Am. Chem. Soc. 104 (1982) 2665.

[27] N. L. Bauld, D. J. Bellville, B. Harirchian, K. T. Lorenz, R. A. Pabon, D. W. Reynolds, D. D. Wirth, H.-S. Chiou, B. K. Marsh, Acc. Chem. Res. 20 (1987) 371.

[28] O. Wiest, E. Steckhan, F. Grein, J. Org. Chem. 57 (1992) 4034.

[29] N. L. Bauld, D. J. Bellville, R. Pabon, R. Chelsky, G. Green, J. Am. Chem. Soc. 105 (1983) 2378.

[30] K. Chockalingam, M. Pinto, N. L. Bauld, J. Am. Chem. Soc. 112 (1990) 447.

[31] G. Buchoux, J.-Y. Salpin, F. Turecek, Rapid Commun. Mass Spectrom. 8 (1994) 325.

[32] M. Hofmann, H. F. Schaefer III, J. Am. Chem. Soc. 121 (1999) 6719.

[33] G. Bouchoux, J.-Y. Salpin, M. Yáñez, J. Phys. Chem. A 108 (2004) 9853. 
[34] Y.-P. Chang, K. Dlugolecki, J. Küpper, D. Rösch, D. Wild, S. Willitsch, Science 342 (2013) 98.

[35] M. N. Eberlin, R. G. Cooks, J. Am. Chem. Soc. 115 (1993) 9226

[36] R. G. Gilbert, S. C. Smith, Theory of unimolecular and recombination reactions, Blackwell Scientific Publications, Oxford, 1990.

[37] Y. Zhao, D. G. Truhlar, Theor. Chem. Acc. 120 (2008) 215

[38] M. J. Frisch, G. W. Trucks, H. B. Schlegel, G. E. Scuseria, M. A. Robb, J. R. Cheeseman, G. Scalmani, V. Barone, B. Mennucci, G. A. Petersson, H. Nakatsuji, M. Caricato, X. Li, H. P. Hratchian, A. F. Izmaylov, J. Bloino, G. Zheng, J. L. Sonnenberg, M. Hada, M. Ehara, K. Toyota, R. Fukuda, J. Hasegawa, M. Ishida, T. Nakajima, Y. Honda, O. Kitao, H. Nakai, T. Vreven, J. A. Montgomery, Jr., J. E. Peralta, F. Ogliaro, M. Bearpark, J. J. Heyd, E. Brothers, K. N. Kudin, V. N. Staroverov, R. Kobayashi, J. Normand, K. Raghavachari, A. Rendell, J. C. Burant, S. S. Iyengar, J. Tomasi, M. Cossi, N. Rega, J. M. Millam, M. Klene, J. E. Knox, J. B. Cross, V. Bakken, C. Adamo, J. Jaramillo, R. Gomperts, R. E. Stratmann, O. Yazyev, A. J. Austin, R. Cammi, C. Pomelli, J. W. Ochterski, R. L. Martin, K. Morokuma, V. G. Zakrzewski, G. A. Voth, P. Salvador, J. J. Dannenberg, S. Dapprich, A. D. Daniels, A. Farkas, J. B. Foresman, J. V. Ortiz, J. Cioslowski, D. J. Fox, Gaussian09 Revision E.01, gaussian Inc. Wallingford CT 2009.

[39] S. Maeda, Y. Harabuchi, Y. Osada, T. Taketsugu, K. Morokuma, K. Ohno, GRRM14 (2013)

[40] S. Maeda, K. Ohno, K. Morokuma, Phys. Chem. Chem. Phys. 15 (2013) 3683.

[41] J. J. P. Stewart, J. Mol. Model. 13 (2007) 1173.

[42] P. J. Stephens, F. J. Devlin, C. F. Chabalowski, M. J. Frisch, J. Phys. Chem. 98 (1994) 11623.

[43] H. P. Hratchian, H. B. Schlegel, J. Chem. Theory Comput. 1 (2005) 61.

[44] F. Aquilante, J. Autschbach, R. K. Carlson, L. F. Chibotaru, M. G. Delcey, L. De Vico, I. F. Galván, N. Ferré, L. M. Frutos, L. Gagliardi, M. Garavelli, A. Giussani, C. E. Hoyer, G. L. Manni, H. Lischka, D. Ma, P. A. Malmqvist, T. Muller, A. Nenov, M. Olivucci, T. B. Pedersen, D. Peng, F. Plasser, B. Pritchard, M. Reiher, I. Rivalta, I. Schapiro, J. SegarraMartí, M. Stenrup, D. G. Truhlar, L. Ungur, A. Valentini, S. Vancoillie, V. Veryazov, V. P. Vysotskiy, O. Weingart, F. Zapata, R. Lindh, J. Comput. Chem. 37 (2016) 506.

[45] J. R. Barker, T. L. Nguyen, J. F. Stanton, C. Aieta, M. Ceotto, F. Gabas, T. J. D. Kumar, C. G. L. Li, L. L. Lohr, A. Maranzana, N. F. Ortiz, J. M. Preses, P. J. Stimac, MultiWell-2016 Software Suite, university of Michigan, Ann Arbor, Michigan, USA, 2016.

[46] W. H. Miller, J. Am. Chem. Soc. 101 (1979) 6810.

[47] Maple 2015.0, maplesoft, a division of Waterloo Maple Inc., Waterloo, Ontario.

[48] E. Hairer, G. Wanner, Solving Ordinary Differential Equations II, 2nd Edition, Springer, New York, 1996.

[49] K. Alder, G. Stein, Justus Liebigs Ann. Chem. 525 (1936) 1.

[50] T. J. Lee, P. R. Taylor, Int. J. Quantum Chem. S23 (1989) 199. 